\title{
Integrated Ontologies for Spatial Scene Descriptions
}

\author{
Sotirios Batsakis \\ Department of Electronic and Computer Engineering \\ Technical University of Crete, Greece \\ Chania, Crete, GREECE \\ batsakis@softnet.tuc.gr \\ Euripides G.M. Petrakis \\ Department of Electronic and Computer Engineering \\ Technical University of Crete, Greece \\ Chania, Crete, GREECE \\ euripides@intelligence.tuc.gr
}

\begin{abstract}
Scene descriptions are typically expressed in natural language texts and are integrated within Web pages, books, newspapers and other means of content dissemination. The capabilities of such means can be enhanced to support automated content processing and communication between people or machines by allowing the scene contents to be extracted and expressed in ontologies, a formal syntax rich in semantics interpretable by both people and machines. Ontologies enable more effective querying, reasoning and general use of content and allow for standardizing the quality and delivery of information across communicating information sources. Ontologies are defined using the well established standards of the Semantic Web for expressing scene descriptions in application fields such as geographic information systems, medicine and the World Wide Web (WWW). Ontologies are not only suitable for describing static scenes with static objects (e.g., in photographs) but also enable representation of dynamic events with objects and properties changing in time (e.g., moving objects in a video). Representation of both static and dynamic scenes by ontologies, as well as querying and reasoning over static and dynamic ontologies are important issues for further research. These are exactly the problems this chapter is dealing with.
\end{abstract}

\section{BACKGROUND}

Formal spatial, temporal and spatio-temporal representations have been studied extensively in the Database (Gutting, 1994) and recently, in the Semantic Web literature (Arpinal, Sheth, Ramakrishnan, Usery, Azami, \& Kwan, 2006). The related work is surveyed in the following sections focusing on work inspired by ontologies and the Semantic Web. 
Spatial entities (e.g., objects, regions) in classic database systems are typically represented based upon a reference coordinate system using points, lines (polygonal lines) or Minimum Bounding Rectangles (MBRs) enclosing objects or regions and their relationships (Petrakis, 2002). Relations among spatial entities can be topologic, orientation or distance relations. In turn, spatial relations can be qualitative (i.e. described using lexical terms) or quantitative (i.e. described using numerical values).

Many spatial ontologies for the semantic Web are known to exist, the majority of them being defined based upon a reference coordinate system and qualitative topologic and direction relations (e.g., RCC-8 relations). Reasoning rules for various relation sets have been proposed as well (Cohn \& Hazarika, 2001), (Renz \& Nebel, 2007). Recent approaches use specialized representation languages such as GML or general purpose Semantic Web languages such as OWL (Abdelmoty, Smart, Jones, Fu, \& Finch, 2005). The SPIRIT spatial search system (Jones, Abdelmoty, Finch, Fu, \& Vaid, 2004) combines an ontology with indexing mechanisms. In (Katz

\& Grau, 2005) RCC-8 topologic relations are represented using OWL-DL. In the work by Hazarika (Hazarika \& Roy, 2008), information retrieval is enhanced using RCC-5 topologic relations among objects. Along the same lines, Grutter (Grutter \& Bauer-Messmer, 2007) suggest extracting RCC-8 relations using a separate coordinate system which is not part of the ontology. SWETO-GS (Arpinal, Sheth, Ramakrishnan, Usery, Azami, \& Kwan, 2006) is a geospatial ontology enhanced with spatiotemporal thematic proximity (STTP) reasoning and interactive visualization capabilities. Liu and Hao (Liu \& Hao, 2005), combine topological and direction relations. A combination of RCC-5 topologic with cardinal direction relations is proposed at (Chen, Liu, Zhang, \& Xie, 2009). An almost orthogonal (to representation) issue is speed of search. Petrakis (Petrakis, 2002a), Dellis and Paliouras (Dellis \& Paliouras, 2007) emphasize on the indexing of spatial information using R-trees for improving the speed of search of nearest-neighbor and range queries.

The representation of spatio-temporal knowledge has also motivated research within the Semantic Web community. Related work includes (Chen, Perich, Finin, \& Joshi, 2004) where the temporal ontology is enhanced by Allen's temporal relations. In this work, the RCC-8 relations form the core of the spatial representation. Worbys and Hornby (Worbys \& Hornby, 2004) suggest a model for representing objects and events combining spatial and temporal information. Wang et.al. (Wang, Zhang, Gu, \& Pung, 2004) introduce a spatiotemporal representation in OWL supporting logic-based reasoning by limiting spatial relations to inclusion relations. In the work by Sheth et.al., (Sheth, Arpinar, Perry, \& Hakimpour, 2009) the SPIRIT spatial query engine (Jones, Abdelmoty, Finch, Fu, \& Vaid, 2004) is combined with temporal RDF (Gutierrez, Hurtado, \& Vaisman, 2007) in an integrated spatiotemporal representation mechanism. Finally, the MOQL query language (Li, Ozsu, Szafron, \& Oria, 1997) has been proposed for querying spatio-temporal information in databases.

\section{INTEGRATED SCENE DESCRIPTION ONTOLOGIES}

Issues relating to spatial and temporal aspects of scene descriptions are discussed next. Particular emphasis in given to ontology models integrating both kinds of knowledge. 


\section{Temporal representation}

Dealing with information that changes over time is a critical problem in Knowledge Representation (KR). Representation languages such as OWL (description logics), frame-based and object-oriented languages (F-logic) are all based on binary relations (e.g., being the employee of a company) without any temporal information. Adding the time dimension to this information would lead to ternary relations which are not supported by OWL (Hayes \& Welty, 2006). However, representation of temporal information using OWL is still feasible, although complicated.

The OWL-Time (formerly DAML-Time) temporal ontology describes the temporal content of Web pages and the temporal properties of Web services. Apart from language constructs for the representation of time in ontologies, there is a need for mechanisms for the representation of the evolution of concepts (events) in time. Existing solutions for dealing with this problem include: Versioning, Reification, the 4-D perdurantist (fluent) approach (Welty \& Fikes, 2006) and Temporal Description Logics (Artale \& Franconi, 2000), (Lutz, Wolter, \& Zakharyaschev, 2008). This is related to the problem of the representation of time in temporal (relational and object oriented) databases (Ozsoyoglu \& Snodgrass, 1995). Existing methods are relying mostly on temporal Entity Relation (ER) models (Gregersen \& Jensen, 1999) taking into account valid time (i.e., the time interval during which a relation holds), transaction time (i.e., the time at which

a database entry is updated) or both. Also, time is represented by time points, intervals or finite sets of intervals. Representation of time in the semantic web standards differs from representation in databases (Gutierrez, Hurtado, \& Vaisman, 2007) as (a) OWL semantics are not equivalent to ER model semantics (e.g., OWL adopts the Open World Assumption) while ER model adopts the Closed World Assumption and (b) relations in OWL are restricted to binary ones.

\section{Ontology Versioning}

Ontology Versioning (Klein \& Fensel) suggests that the ontology has different versions (one per instance of time). When a change takes place, a new version is created. Versioning suffers from several disadvantages: (a) changes even on single attributes require that a new version of the ontology be created leading to information redundancy (b) searching for events occurred at time instances or during time intervals requires exhaustive searches in multiple versions of the ontology and, (c) it is not clear how the relation between evolving classes is represented within the ontology.

\section{Temporal Description Logics}

Temporal description logics (Artale \& Franconi, 2000) (Lutz, Wolter, \& Zakharyaschev, 2008) form the basis of Semantic Web standards such as OWL-DL and are based on Description Logics (DL). In addition to the features of standard DLs, Temporal DLs allow additional operators such as "until", "sometime in the past" and "always in the future". They extend the expressive power of standard description logics and they don't suffer from the data redundancy problem of other approaches (versioning, 4-D fluent, and reification). They also require extending standard Semantic Web languages such as OWL, which is not desirable when compatibility with existing standards and tools is required. 


\section{Reification}

Reification (Andronikos, Stefanidakis, \& Papadakis, 2009) is a general purpose technique applied when relations involving more than two objects-attributes (such as temporal relations) have to be represented using languages restricting relations to binary ones. For example when the relation Rel holds between objects $O b j_{1}$ and $O b j_{2}$ at time time $e_{1}$ then a new object is created which has three properties: Objects $O b j_{1}$ and $O b j_{2}$ and the time value time $e_{1}$. Data redundancy is a problem of this method, since a new object is created whenever a temporal relation must be represented. In addition, since relations are represented as objects and not as OWL relations, the semantics offered by the OWL specification regarding object relations can't be applied when reification is used.

\section{The 4-Dimensional (Perdurantist) Approach}

The 4D-fluent (perdurantist) approach (Welty \& Fikes, 2006) shows how temporal information can be represented effectively in OWL. However, similarly to versioning and reification, it still suffers from data redundancy. Concepts in time are represented as 4-dimensional objects with the 4th dimension being the time. Time instances and time intervals are represented as instances of a time interval class which in turn, is related with time concepts varying in time.

The ontology comprises of a static and a dynamic part. Changes occur on the properties of the temporal part of the ontology keeping the entities of the static part unchanged. To add the time dimension to an ontology, classes TimeSlice and TimeInterval with properties tsTimeSliceOf and tsTimeInterval respectively are introduced into the ontology representation. Class TimeSlice is the domain class for entities representing temporal parts (i.e., "time slices") and class TimeInterval is the domain class of time intervals. A time interval holds the temporal information of a time slice. Property tsTimeSliceOf connects an instance of class TimeSlice with an entity, and property tsTimeInterval connects an instance of class TimeSlice with an instance of class TimeInterval. Properties having a time dimension are called fluent properties and connect instances of class TimeSlice.

Figure 1 illustrates an example temporal ontology with classes Company with data type property companyName and Product with datatype properties price and productName. In this example, CompanyName is a static property (its value does not change in time), while properties produces, productName and price are dynamic (fluent) properties whose values may change in time. Because they are fluent properties, their domain (and range) is of class TimeSlice. CompanyTimeSlice and ProductTimeSlice are instances of class TimeSlice and are provided to denote that the domain of properties produces, productName and price are time slices restricted to be slices of a specific class. For example, the domain of property productName is not class TimeSlice but it is restricted to instances that are time slices of class Product. 

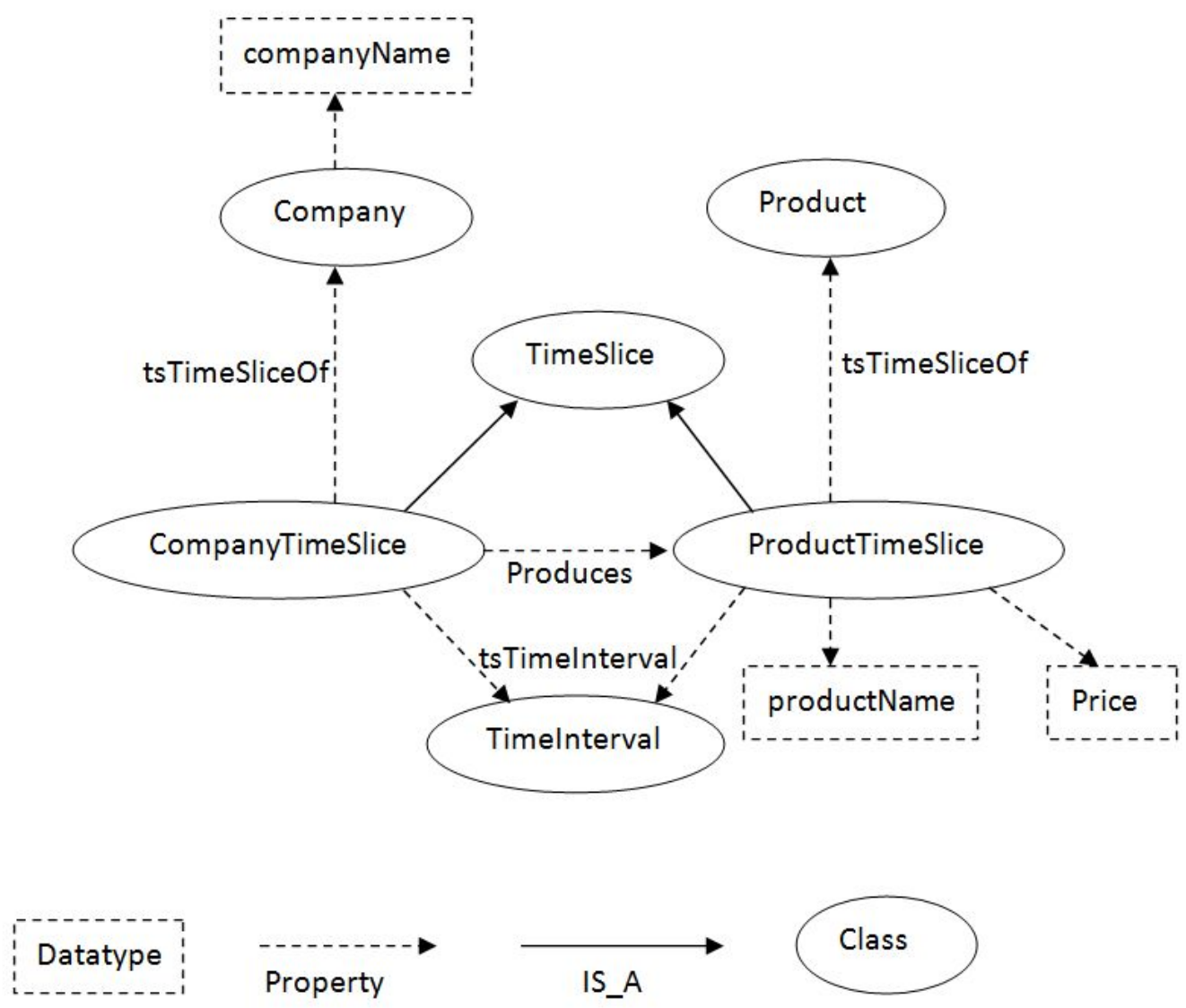

Figure 1.Ontology for representation of dynamic objects using 4D fluents.

\section{Spatio-Temporal Representations}

Representing spatial knowledge has been studied extensively within the context of spatial database research (Gutting, 1994). Existing representations refer mostly to quantitative data (i.e., points, lines and minimum bounding rectangles). Quantitative representation mechanisms are best suited to Geographical Information systems (GIS). Spatial relations holding between objects can be topologic, directional and distance relations. Qualitative relations although less accurate than quantitative relations have been proposed as well. The vast majority of scene descriptions expressed in natural language involve mostly qualitative expressions which are more common than quantitative ones. For instance, the relative position between two regions is commonly expressed by qualitative expressions such as "North of" rather than by quantitative ones, such as by providing longitude and latitude values. Quantitative descriptions are restricted to specialized domains (such as navigation in GIS) while, in everyday use qualitative relations are more common.

Similarly to spatial relations, temporal relations (e.g., "before”, "after”) often appear without numerical values denoting time instants or intervals. Integrating temporal and spatial representations in a unified model has been studied in (Abraham \& Roddick, 1999) and (Sheth, Arpinar, Perry, \& Hakimpour, 2009) in the context of database and semantic Web research 
respectively. Integrating spatial with temporal representations in a unified ontology representation model is discussed next.

\section{Spatial Ontology Representation}

The 4-D fluent mechanism will form the basis of the proposed spatio-temporal ontology representation. The spatial representation mechanism below supports several types of qualitative spatial relations (topologic, directional, distance). Although many applications require only one category of relations, a general purpose ontology model should offer support for all types of spatial relations.

Figure 2 illustrates a general ontology representation model for spatial information. Class Location has attribute name (of type string). Also a Location object can be optionally connected with a footprint class object with subclasses: Point, Line, Polyline and MBR. Class Point has two (or three in a three-dimensional representation) numerical attributes, namely $X, Y$ (also $Z$ in a three-dimensional representation). For example, Point will be the footprint of entities such as cities in a large scale map. Class Line has point ${ }_{1}$ and point ${ }_{2}$ as attributes representing the ending points of a line segment. Class PolyLine may represent region boundaries by a set of consecutive line segments (e.g. roads, rivers). Finally, a region can be represented by the polygonal line surrounding its contour (Polyline) or by its Minimum Bounding Rectangle (MBR). In the later case, the MBR of a region is denoted by four numerical attributes Xmax, YmaxY, Xmin and Ymin. As Figure 2 demonstrates, both Polyline and MBR attributes can be taking part in the same representation.

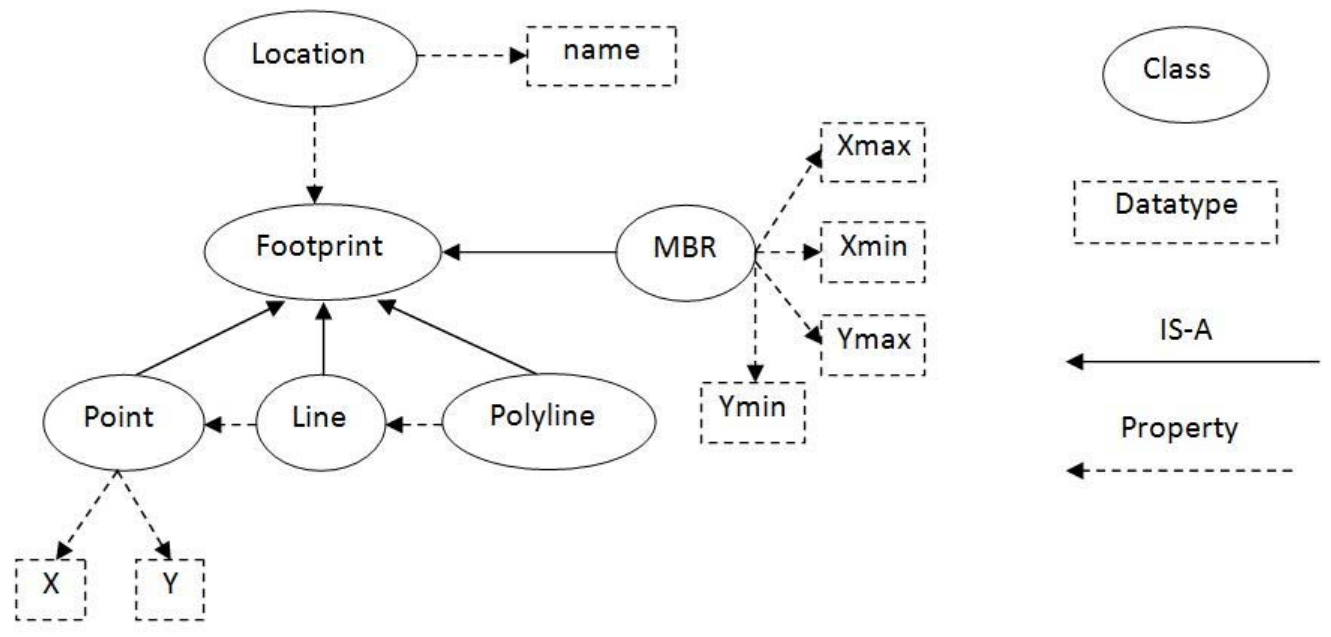

Figure 2. Ontology representation of spatial objects.

Figure 3 summarizes all types of spatial relations within a common ontology schema. Omitting one or more types of spatial relations is a design decision which is taken given application domain and user requirements. 


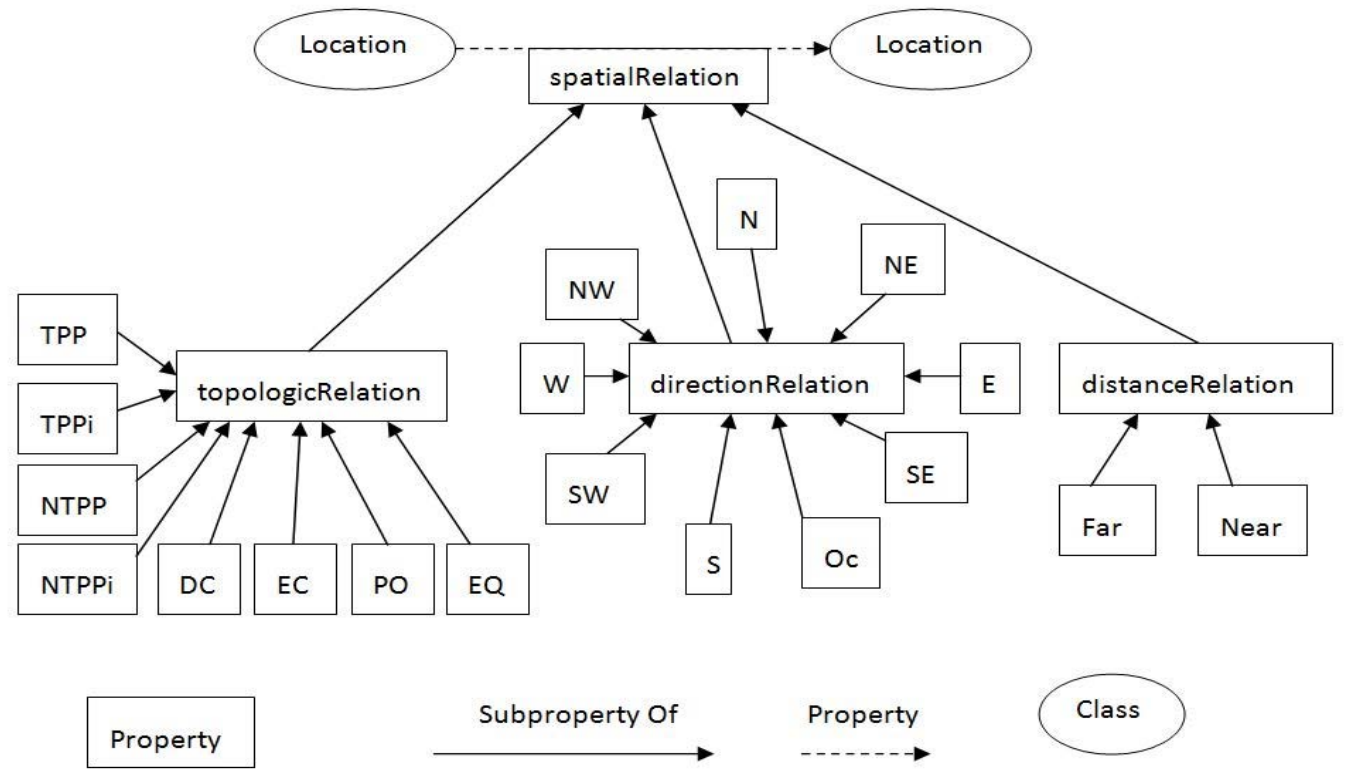

Figure 3. Ontology schema with all spatial relations.

The spatial relations between regions can be easily extracted from their surrounding MBRs (or surrounding polygonal lines) by comparing their coordinates. In an ontology, each spatialRelation connects two locations and has three subproperties namely: topologicRelation, directionalRelation and distanceRelation. These types of relations may co-exist within the same representation although in most cases, depending on the application, an ontology schema calls for either direction or topologic relations. The following topologic relations (DC, EC, EQ, NTTP, NTTPi, TTP, TPPi, PO), shown in Figure 4, referred to as RCC-8 relations (Randell, Cui, \& Cohn, 1992) are also defined and can be used as well:
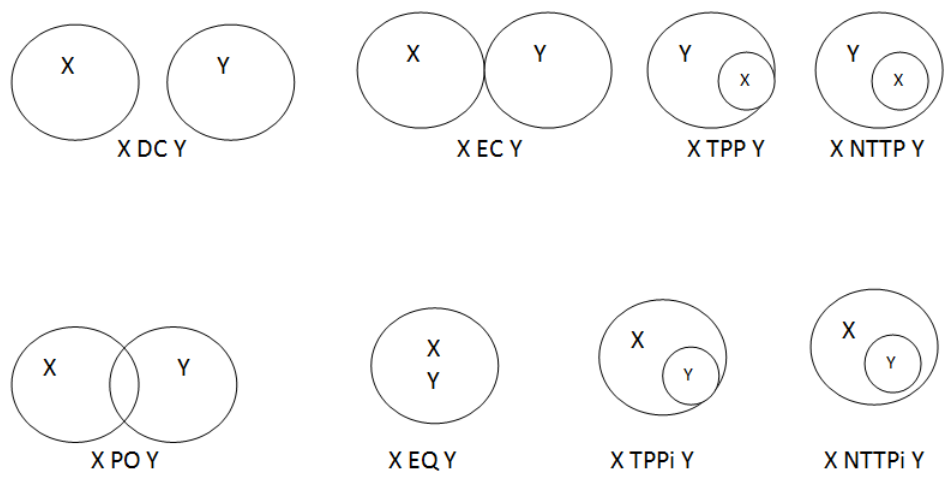

Figure 4. RCC-8 topologic relations.

Direction relations are defined based on 2D or cone shaped projections (Renz \& Nebel, 2007). As shown in Figure 5, nine direction relations can be identified namely North (N), North East (NE), East (E), South East (SE), South (S), South West (SW), West (W), North West (NW) and the neutral direction (Oc) based on 2D-projections (Frank, 1996). In addition, the relations Front, Left, Behind and Right can also be used. 


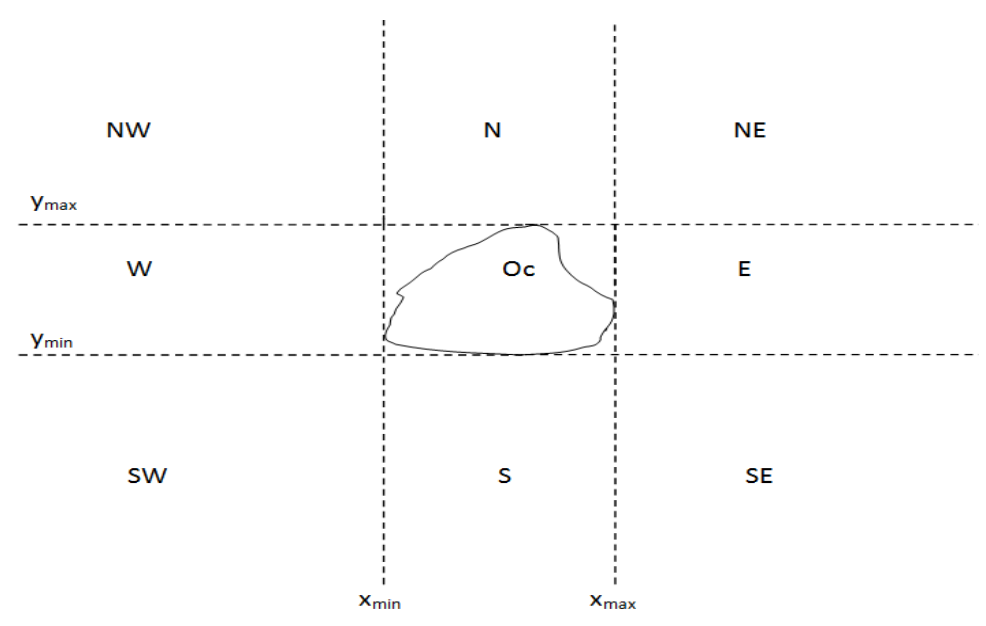

Figure 5.Projection based direction relations.

Finally, the distance relations far and near can be defined. However, distance relations may be ambiguous especially in application where a common scale for measuring distances is not provided. Also, distance relations can be expressed quantitatively (e.g., 3Km far from city A) and stored in the ontology using reification (i.e., by defining a distance object with attributes the two related locations and a numerical attribute representing distance).

Summarizing, it is likely that only topological and direction relations will be used in practice since they can be easily extracted from natural scenes. Distance relations, although common in natural scene descriptions, are less likely to be used since they cannot be extracted from natural scenes (unless a reference coordinate system or scale for measuring distances is provided). For instance, given the MBRs of two regions, the EQ relation holds when all four coordinates are equal, $D C$ relation holds when either $x$-axis or $y$-axis coordinates -or both- do not overlap (i.e., the min value of a region is greater than the corresponding maximum value of the other) etc. Additional relations can be inferred by the defined ones using composition tables which are defined for both topologic and direction relations.

A composition table defines the possible spatial relations holding between two objects, given their spatial relations with a third one. Table 1 and Table 2 illustrate example composition tables using RCC-8 topologic and 2D projection based direction relations respectively. Spatial reasoning is then achieved by applying rules implementing the inferred relations of a composition table. 


\begin{tabular}{|c|c|c|c|c|c|c|c|c|}
\hline & DC & EC & $\mathrm{PO}$ & TPP & NTPP & TPPi & NTPPi & EQ \\
\hline DC & $\begin{array}{l}\text { DC,EC,PO, } \\
\text { TPP,NTPP, } \\
\text { TPPi,NTPPi, } \\
\text { EQ }\end{array}$ & $\begin{array}{l}\text { DC,EC,PO, } \\
\text { TPP,NTPP }\end{array}$ & $\begin{array}{l}\text { DC,EC,PO, } \\
\text { TPP,NTPP }\end{array}$ & $\begin{array}{l}\text { DC,EC,PO, } \\
\text { TPP,NTPP }\end{array}$ & $\begin{array}{l}\text { DC,EC,PO, } \\
\text { TPP,NTPP }\end{array}$ & DC & $\mathrm{DC}$ & $\mathrm{DC}$ \\
\hline EC & $\begin{array}{l}\text { DC,EC,PO, } \\
\text { TPPi,NTPPi }\end{array}$ & $\begin{array}{c}\text { DC,EC,PO,TPP } \\
\text { TPPi,EQ } \\
\end{array}$ & $\begin{array}{l}\text { DC,EC,PO, } \\
\text { TPP,NTPP }\end{array}$ & $\begin{array}{c}\text { EC,PO, } \\
\text { TPP,NTPP }\end{array}$ & PO,TPP,NTPP & $\mathrm{DC}, \mathrm{EC}$ & DC & EC \\
\hline PO & $\begin{array}{l}\text { DC,EC,PO, } \\
\text { TPPi,NTPPi }\end{array}$ & $\begin{array}{l}\text { DC,EC,PO, } \\
\text { TPPi,NTPPi }\end{array}$ & $\begin{array}{c}\text { DC,EC,PO,TPP, } \\
\text { NTPP,TPPi, } \\
\text { NTPPi,EQ } \\
\end{array}$ & PO,TPP,NTPP & PO,TPP,NTPP & $\begin{array}{l}\text { DC,EC,PO, } \\
\text { TPPi,NTPPi }\end{array}$ & $\begin{array}{l}\text { DC,EC,PO, } \\
\text { TPPi,NTPPi }\end{array}$ & PO \\
\hline TPP & $\mathrm{DC}$ & $\mathrm{DC}, \mathrm{EC}$ & $\begin{array}{l}\text { DC,EC,PO, } \\
\text { TPP,NTPP }\end{array}$ & TPP,NTPP & NTPP & $\begin{array}{l}\text { DC,EC,PO, } \\
\text { TPP,NTPP }\end{array}$ & $\begin{array}{l}\text { DC,EC,PO, } \\
\text { TPPi,NTPPi }\end{array}$ & TPP \\
\hline NTPP & DC & DC & $\begin{array}{l}\text { DC,EC,PO, } \\
\text { TPP,NTPP }\end{array}$ & NTPP & NTPP & $\begin{array}{l}\text { DC,EC,PO, } \\
\text { TPP,NTPP }\end{array}$ & $\begin{array}{c}\text { DC,EC,PO } \\
\text { TPP,NTPP, } \\
\text { TPPi,NTPPi } \\
\text { EQ } \\
\end{array}$ & NTPP \\
\hline TPPi & $\begin{array}{l}\text { DC,EC,PO, } \\
\text { TPPi,NTPPi }\end{array}$ & $\begin{array}{c}\text { EC,PO, } \\
\text { TPPi,NTPPi }\end{array}$ & $\begin{array}{l}\text { PO,TPPi, } \\
\text { NTPPi }\end{array}$ & $\begin{array}{c}\text { EQ,PO,TPPi, } \\
\text { NTPPi }\end{array}$ & PO,TPP,NTPP & TPPi,NTPPi & NTPPi & TPPi \\
\hline NTPPi & $\begin{array}{l}\text { DC,EC,PO, } \\
\text { TPPi,NTPPi }\end{array}$ & $\begin{array}{l}\text { PO,TPPi, } \\
\text { NTPPi }\end{array}$ & $\begin{array}{l}\text { PO,TPPi, } \\
\text { NTPPi }\end{array}$ & $\begin{array}{c}\text { PO,TPPi, } \\
\text { NTPPi }\end{array}$ & $\begin{array}{l}\text { PO,TPP,NTPP } \\
\text { EQ,TPPi,NTPPi }\end{array}$ & NTPPi & NTPPi & NTPPi \\
\hline EQ & DC & $\mathrm{EC}$ & $\mathrm{PO}$ & TPP & NTPP & TPPi & NTPPi & EQ \\
\hline
\end{tabular}

Table 1. Composition of RCC-8 topologic relations.

\begin{tabular}{|c|c|c|c|c|c|c|c|c|c|}
\hline & $\mathrm{N}$ & $\mathrm{NE}$ & $\mathrm{E}$ & SE & $\mathrm{s}$ & SW & W & NW & Oc \\
\hline $\mathrm{N}$ & $\mathrm{N}$ & $\mathrm{N}$ & NW & $\begin{array}{c}\mathrm{E}, \mathrm{NE}, \mathrm{SE}, \mathrm{Oc}, \mathrm{N}, \\
\mathrm{S}\end{array}$ & $\mathrm{Oc}, \mathrm{N}, \mathrm{S}$ & $\begin{array}{c}\mathrm{W}, \mathrm{NW}, \mathrm{SW} \\
\mathrm{N}, \mathrm{S}, \mathrm{Oc}\end{array}$ & NW & NW & $\mathrm{N}$ \\
\hline NE & $\mathrm{NE}$ & $\mathrm{NE}$ & $\mathrm{NE}$ & $\mathrm{E}, \mathrm{NE}, \mathrm{SE}$ & $\begin{array}{l}\mathrm{E}, \mathrm{NE}, \\
\mathrm{SE}\end{array}$ & $\begin{array}{c}\text { Oc,S,SE,SW, } \\
\text { W,NW, } \\
\text { N,NE,E }\end{array}$ & $\begin{array}{l}\text { N,NE, } \\
\text { NW }\end{array}$ & $\mathrm{N}, \mathrm{NE}, \mathrm{NW}$ & $\mathrm{NE}$ \\
\hline $\mathrm{E}$ & $\mathrm{NE}$ & $\mathrm{NE}$ & $\mathrm{S}$ & SE & SE & $\begin{array}{l}\text { S,SW,SE } \\
\mathrm{Oc}, \mathrm{E}, \mathrm{W},\end{array}$ & $\mathrm{Oc}, \mathrm{E}, \mathrm{W}$ & $\begin{array}{c}\mathrm{N}, \mathrm{NW}, \mathrm{NE}, \\
\mathrm{Oc}, \mathrm{E}, \mathrm{W}\end{array}$ & $\mathrm{E}$ \\
\hline SE & $\begin{array}{c}\text { E,SE, } \\
\mathrm{NE}\end{array}$ & $\mathrm{E}, \mathrm{SE}, \mathrm{NE}$ & SE & SE & SE & $\mathrm{S}, \mathrm{SE}, \mathrm{SW}$ & S,SE,SW & $\begin{array}{c}\text { Oc,S,SE,SW,W } \\
N W, N, N E, E\end{array}$ & SE \\
\hline s & $\mathrm{Oc}, \mathrm{S}, \mathrm{N}$ & $\begin{array}{c}\mathrm{E}, \mathrm{S}, \mathrm{N}, \mathrm{Oc}, \mathrm{NE}, \\
\mathrm{SE}\end{array}$ & SE & SE & $\mathrm{s}$ & SW & SW & $\begin{array}{c}\mathrm{W}, \mathrm{S}, \mathrm{N}, \mathrm{Oc}, \mathrm{NW}, \\
\text { SW }\end{array}$ & s \\
\hline sw & $\begin{array}{l}\text { W,SW } \\
\text { NW }\end{array}$ & $\begin{array}{c}\text { Oc,S,SE,SW, } \\
\text { W,NW,N, } \\
\text { NE,E }\end{array}$ & $\begin{array}{l}\text { S,SW } \\
\text { SE }\end{array}$ & S,SW,SE & SW & SW & SW & W,NW,SW & SW \\
\hline w & NW & $\begin{array}{c}\mathrm{N}, \mathrm{NW}, \mathrm{NE} \\
\mathrm{Oc}, \mathrm{W}, \mathrm{E}\end{array}$ & $\mathrm{Oc}, \mathrm{W}, \mathrm{E}$ & $\begin{array}{r}\mathrm{S}, \mathrm{SE}, \mathrm{SW} \\
\mathrm{Oc} \mathrm{E}, \mathrm{W} \\
\end{array}$ & sW & SW & W & NW & W \\
\hline NW & NW & $\mathrm{N}, \mathrm{NW}, \mathrm{NE}$ & $\begin{array}{l}\mathrm{N}, \mathrm{NW} \\
\mathrm{NE}\end{array}$ & $\begin{array}{c}\mathrm{Oc}, \mathrm{S}, \mathrm{SE}, \mathrm{SW}, \\
\mathrm{W}, \mathrm{NW}, \mathrm{N}, \\
\mathrm{NE}, \mathrm{E}\end{array}$ & $\begin{array}{l}\text { W,NW, } \\
\text { SW }\end{array}$ & W,NW,SW & NW & NW & NW \\
\hline Oc & $\mathrm{N}$ & $\mathrm{NE}$ & $\mathrm{E}$ & SE & $\mathrm{S}$ & SW & W & NW & Oc \\
\hline
\end{tabular}

Table 2. Composition of $2 \mathrm{D}$ projection based direction relations.

A limited set of table entries leads to an unambiguously defined result. For example, the composition of the S and SE direction relations (i.e., object A is south of B and object B southeast of $\mathrm{C}$ ) yields (as defined by the table entry at the intersection of the $S$-row and the $S E$-column of Table 2) the $S E$ relation as a result meaning that A is south east of C. However, the composition of $E$ and $N W$ relations doesn't yield a unique relation as a result. Therefore only a subset of the above table entries (27 in the case of RCC-8 relations and 49 in the case of projection based direction relations) can be used to infer other relations. If the directional relations "front", "left", "right" and "behind" are used then, the corresponding reasoning mechanism has to be used (Freksa, 1992). In this case determining the relation between two objects A and B doesn't determine the inverse relation holding between object B and A. 


\section{Spatio-Temporal Ontology Representation}

In the following, we show how spatial representations are combined with the prevailing temporal representation presented in the previous sections (the 4D fluent approach) into an integrated spatio-temporal ontology model. For the ease of presentation we distinguish between application cases involving static or moving objects.

\section{Static objects}

Figure 6 illustrates a static object (i.e. objects without fluent properties) which is related with the hasLocation property with a Location object, which in turn is related with a footprint (Figure 2) or with other locations using user defined or footprint extracted relations (Figure 3).

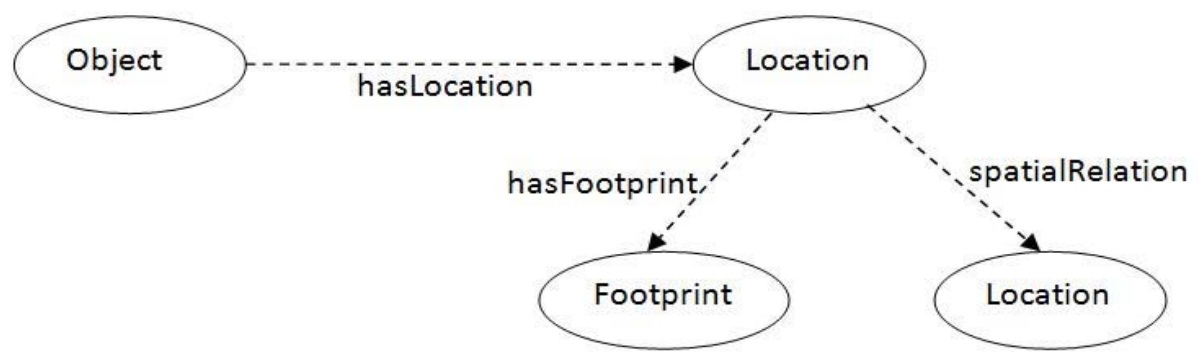

Figure 6.Static objects.

\section{Dynamic objects}

In the case of dynamic objects whose location is static (i.e., although the objects don't change location, other properties of the object, e.g., the color of a house, may change) the location property must be defined at the static part of the ontology, as illustrated in Figure 7. Note that, the location of an object is represented using a single entry although multiple time slices of this object may exist.

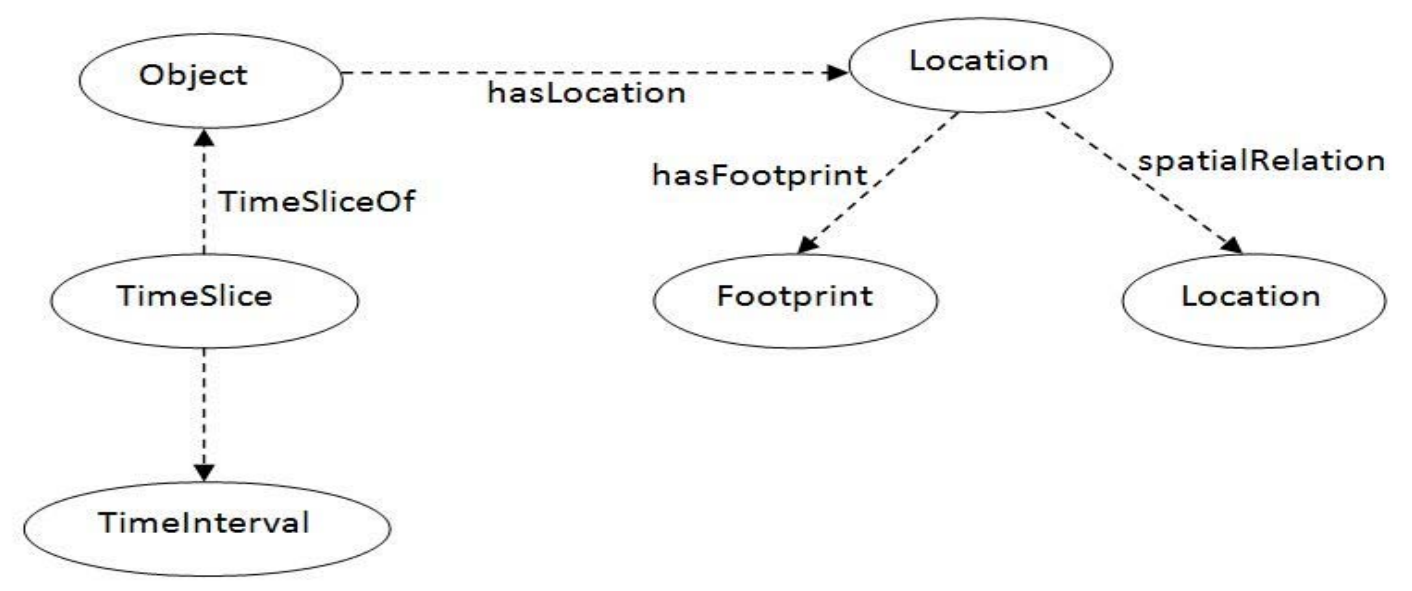

Figure 7.Dynamic objects with static position. 
In the case of moving objects, the hasLocation property applies to the TimeSlice class and not to the static object. It is also related to a corresponding time interval. This is illustrated in Figure 7. This is the most complicated case and refers to moving objects (i.e., objects whose position change) or events that evolve in space and time (i.e., taking place in different locations as time evolves).

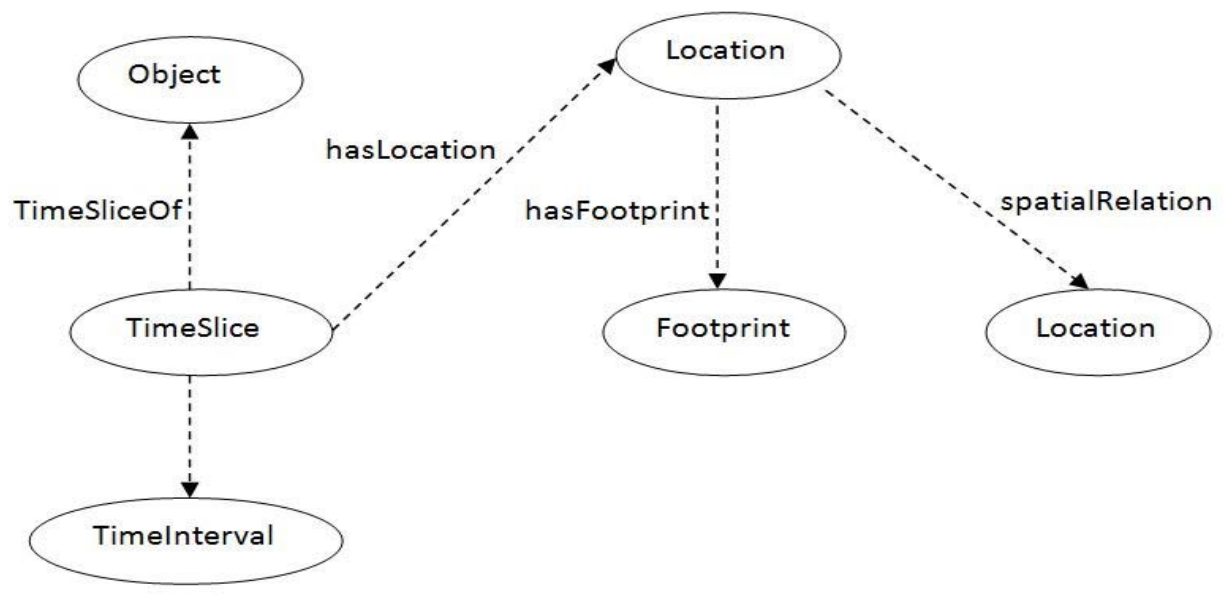

Figure 8.Dynamic moving Objects.

Fig. 9 illustrates the dynamic ontology schema representing the scenario "... from the city of Chania, John went to the city of Athens located further north”.

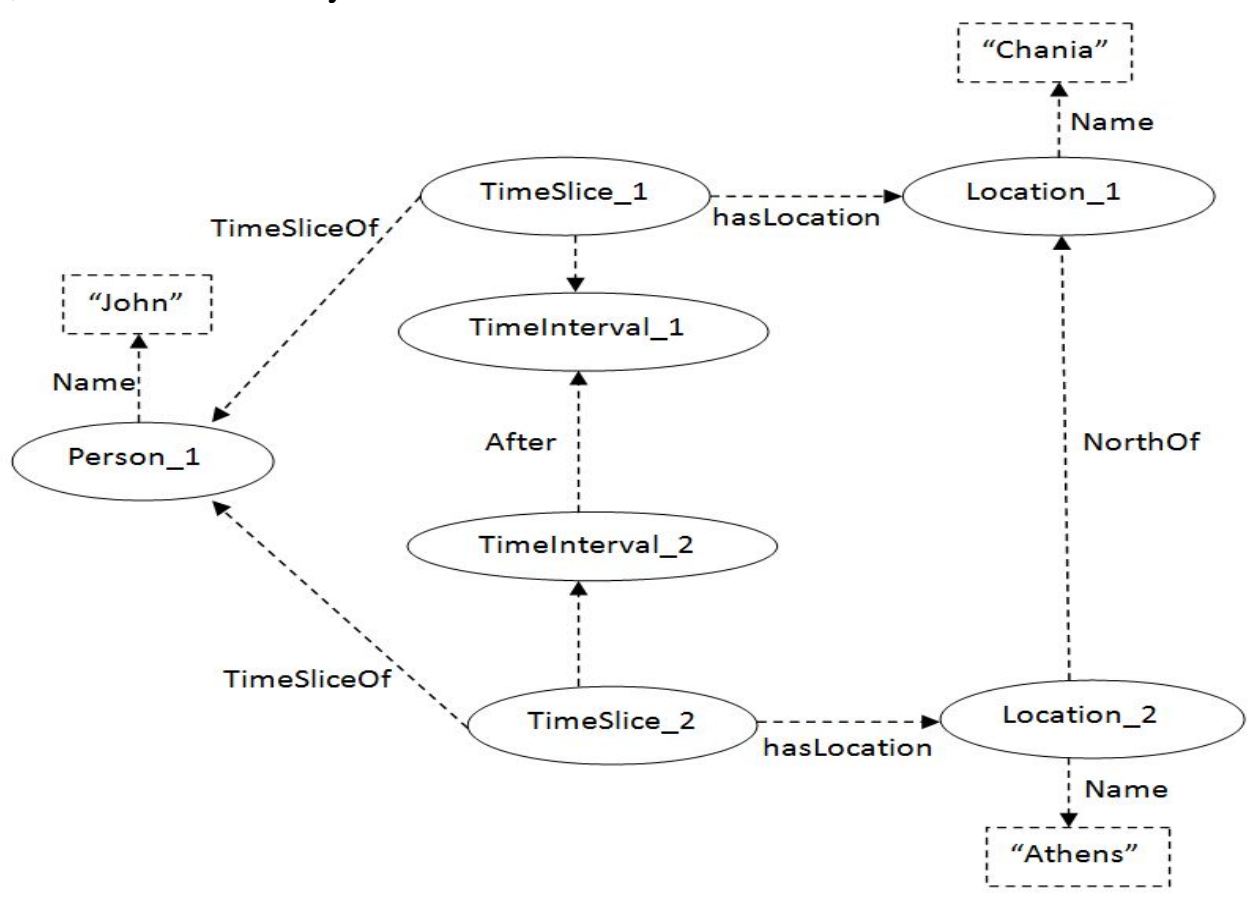

Figure 9.Instantiation example. 
In the above representation the following temporal semantics hold:

- A spatial relation between two static objects holds at all times (e.g. the direction relation between two cities)

- A spatial relation between the location of a static object and a location of a timeslice object, holds for the time interval the time-slice holds true (e.g., if a ship is at a port for a given time interval then the inclusion topologic relation between the ship and the port holds for that time interval only).

- A spatial relation between the locations of two timeslices holds as long as both timeslices hold true (i.e., for the time defined by the intersection of their corresponding time intervals, e.g., being passenger in a ship).

The far and near relations can be included in the representation model as user defined properties (i.e., the user can manually assert that the near or far relation holds between two objects). However, because they are application and scale dependent, automatic extraction is not suitable for a general purpose representation. The point and MBR based representation can be used in the ontology as well, although specific applications might require more accurate location representations based on polygonal approximations of objects or regions (which however are more complicated than MBRs as the algorithms for extracting and handling such representations are computationally more involved).

\section{FUTURE RESEARCH DIRECTIONS}

Ontology representations of dynamic scene descriptions can be realized using a combination of existing temporal and spatial representations mechanisms. Crafting and instantiating such dynamic ontologies manually can be tedious and time consuming tasks that can be carried out only by domain experts. Instantiating information to a spatio-temporal ontology usually calls for application of information extraction (ACE, 2007) or text mining approaches (Camiano, 2006) for the extraction or recognition of concepts, events and locations (as the majority of information residing in information sources such as the Web is in textual form). Querying spatio-temporal information in ontologies using general purpose Semantic Web query languages such as SPARQL and SeRQL leads to formation of complicated queries. Developing ontology query languages (or extending existing languages) for handling spatio-temporal information in ontologies is a direction of promising research (Baratis, Petrakis, Batsakis, Maris, \& Papadakis, 2009).

\section{CONCLUSION}

In this chapter, ontology approaches for representing static and dynamic scene contents are discussed. Example representations based on the 4-D fluent temporal representation mechanism combined with topologic RCC-8 and projection based 2D directional relations are presented. The resulting ontology can be instantiated using both qualitative and quantitative information. Different types of temporal and spatial representations are all integrated into a unique spatiotemporal ontology representation capable of representing static, temporal and both or combined 
spatio-temporal information. The proposed representation is a general purpose one, and is capable of representing temporal and spatial aspects of the events and of the objects involved in a scene.

\section{REFERENCES}

Abdelmoty, A., Smart, P., Jones, C., Fu, G., \& Finch, D. (2005). A critical evaluation of ontology languages for geographic information retrieval on the Internet. Journal of Visual Languages \& Computing, 16(4), pp. 331-358.

Abraham, T., \& Roddick, J. (1999). Survey of spatio-temporal databases.

GeoInformatica, 3(1), pp. 61-99.

ACE. (2007). The ACE Evaluation Plan: Evaluation of the Detection and Recognition of ACE Entities, Values, Temporal Expressions, Relations and Events, ver. 1.9, http://www.nist.gov/speech/tests/ace/2007/index.html.

Andronikos, T., Stefanidakis, M., \& Papadakis, I. (2009). Adding Temporal Dimension to Ontologies via OWL Reification. Proceedings of Panhellenic Conference on Informatics , pp. 19-22.

Arpinal, B., Sheth, A., Ramakrishnan, C., Usery, L., Azami, M., \& Kwan, M.-P. (2006). Geospatial ontology development and semantic analytics. Transactions in GIS, 10(14), pp. 551-575.

Artale, A., \& Franconi, E. (2000). A survey of temporal extensions of description logics. Annals of Mathematics and Arti cial Intelligence, 30(1-4) , pp. 171-210.

Baratis, E., Petrakis, E. G., Batsakis, S., Maris, N., \& Papadakis, N. (2009, July 8-10). TOQL: Temporal Ontology Querying Language. 11th International Symposium on Spatial and Temporal Databases (SSTD 2009), pp. 338-354.

Camiano, P. (2006). Ontology Learning and Population from Text: Algorithms, Evaluation and Applications-Springer.

Chen, H., Perich, F., Finin, T., \& Joshi, A. (2004). Soupa: Standard ontology for ubiquitous and pervasive applications. Proc. of International Conference on Mobile and Ubiquitous multimedia (MOBIQUITOUS), Baltimore, MD , pp. 258-267.

Chen, J., Liu, D., Zhang, C., \& Xie, Q. (2009). Combinative Reasoning with RCC5 and Cardinal Direction Relations. Lecture Notes in Computer Science, Springer Berlin / Heidelberg, Vol. 4798/2009, pp. 92-102.

Cohn, A., \& Hazarika, S. (2001). Qualitative Spatial Representation and Reasoning: An Overview. Lecture Notes in Computer Science, Springer Berlin/Heidelberg, Qualitative Spatial Reasoning with Topological Information, Vol 2293/2002 , pp. 179-182. 
Dellis, E., \& Paliouras, G. (2007). Management of large spatial ontology bases. Lecture Notes in Computer Science Springer Verlag, Ontology-Based Databases and Information Systems, Vol 4623/2007, pp. 102-118.

Frank, A. (1996). Qualitative Spatial Reasoning:Cardinal Directions as an Example. International Journal of Geographic Information Systems. 10(3) , pp. 269-290.

Freksa, C. (1992). Using orientation information for qualitative spatial reasoning. Theories and Methods of Spatio-Temporal Reasoning in Geographic Space, Springer, Volume 639/1992, pp. 162-178.

Gregersen, H., \& Jensen, C. (1999). Temporal Entity-Relationship models-A survey. IEEE Transactions on Knowledge and Data Engineering, 11(3) , pp. 464-497.

Grutter, R., \& Bauer-Messmer, B. (2007). Towards spatial reasoning in the semantic web: A hybrid knowledge representation system architecture. Lecture Notes in Geoinformation and Cartography - Springer Berlin/Heidelberg, The European Information Society. Leading the Way with Geo-Information. Part 8 , pp. 349-364.

Gutierrez, C., Hurtado, C., \& Vaisman, A. (2007). Introducing time into RDF. IEEE Transactions on Knowledge and Data Engineering, 19(2) , pp. 207-218.

Gutting, R. (1994). An introduction to Spatial Database Systems. VLDB Journal, 3 , pp. 357-399.

Hayes, P., \& Welty, C. (2006). Defining N-ary Relations on the Semantic Web. W3C Working Group, http://www.w3.org/TR/swbp--n-aryRelations/ .

Hazarika, J., \& Roy, B. (2008). Semantic Search of Unstructured Knowledge using Qualitative Analysis. International Journal of Knowledge Management, 4(2) IGI Global , pp. 35-45.

Jones, C., Abdelmoty, A., Finch, D., Fu, G., \& Vaid, S. (2004). The SPIRIT search engine: Architecture, Ontologies and Spatial Indexing. Lecure Notes in Computer Science, Springer Berlin/Heidelberg, Vol. 3234/2004 .

Katz, Y., \& Grau, B. (2005). Representing qualitative spatial information in OWL-DL. Proceedings of Intern. Workshop: OWL Experiences and Directions, Galway, Ireland.

Klein, M., \& Fensel, D. (n.d.). Ontology Versioning for the Semantic Web. Proceedings of Intern. Semantic Web Working Symposioum (SWWS' 01), California, USA , pp. 75-92. Li, J., Ozsu, M., Szafron, D., \& Oria, V. (1997). MOQL: A multimedia object query language. Proceedings of the 3rd International Workshop on Multimedia Information Systems, Como, Italy , pp. 19-28. 
Liu, Y., \& Hao, Z. (2005). The cardinal direction relations and the rectangle algebra. Proc. of Intern. Workshop on Machine Learning and Cybernetics, 8, Guangzhou, China , pp. 3115-3118.

Lutz, C., Wolter, F., \& Zakharyaschev, M. (2008). Temporal description logics: A survey. In Proc. of Intern. Conference on Temporal Representation and Reasoning (TIME08), Montreal, QC , pp. 3-14.

Ozsoyoglu, G., \& Snodgrass, R. (1995). Temporal and Real-Time databases: A Survey. IEEE Transactions on Knowledge and Data Engineering 7(4) , pp. 513-532.

Petrakis, E. G. (2002a). Design and Evaluation of Spatial Similarity Approaches for Image Retrieval. Image and Vision Computing,20(1) , pp. 59-76.

Petrakis, E. G. (2002). Fast Retrieval by Spatial Structure in Image Databases. Journal of Visual Languages and Computing, 13(5) , pp. 545-569.

Randell, D., Cui, Z., \& Cohn, A. (1992). A spatial logic based on regions and connection. Proc. 3rd Int. Conf. on Knowledge Representation and Reasoning, Morgan Kaufmann, San Mateo , pp. 165-176.

Renz, J., \& Nebel, B. (2007). Qualitative Spatial Reasoning using Constraint Calculi. Handbook of Spatial Logics, Springer, Netherlands , pp. 161-215.

Sheth, A., Arpinar, I., Perry, M., \& Hakimpour, F. (2009). Geospatial and Temporal Semantic Analytics. Handbook of Research in Geoinformatics, Hassan A. Karimi (eds), Chapter XXI .

Wang, X., Zhang, D., Gu, T., \& Pung, H. (2004). Ontology based context modeling and reasoning using OWL. Proceedings of the Second IEEE conference on Pervasive Computing and Communications Workshops, Orlando Florida, USA .

Welty, C., \& Fikes, R. (2006). A Reusable Ontology for Fluents in OWL. Proceedings of Intern. Conference on Formal Ontology in Information Systems (FOIS-2006). Baltimore, USA , pp. 226-236.

Worbys, M., \& Hornby, K. (2004). From Objects to Events: GEM, the geospatial event model. Proc. of Intern. Conference on GIScience 2004, Lecture Notes in Computer Science, Vol. 3234, Springer Verlag, pp. 327-344.

\section{KEY TERMS \& DEFINITIONS}

Semantic Web, Ontology, Temporal Ontology, Spatial Ontology, SpatioTemporal Ontology, Temporal Representation, Spatial Representation, Qualitative Spatial Relations, Topologic Relations, Direction Relations, Reasoning. 